www.conferenceie.ase.ro

\title{
MOBILE SYSTEMS SECURITY
}

\author{
Ioan ADASCALITEI \\ Bucharest University of Economic Studies, Romania \\ ioan.adascalitei@ie.ase.ro
}

\begin{abstract}
Mobile devices threats are all over and come in many ways. Nowadays the number of mobile devices is extremely high, and we use them to cover many personal needs like ordering food, buying plane tickets, controlling our home from distance (using IoT devices) and so on. But all of this comes with a cost and that cost is that we have to give personal data to some devices which lead to threats. This paper is an overview of what we know so far about threats which affects the mobile devices at system and physical level, how they work and their scope. A classification of them is made so we can define them better in order to find ways for preventing and why not extinguish so we can be safer. As method of research was used a qualitative one by documenting from articles related to this theme, reports realized by companies which operates in this field and other resources.
\end{abstract}

Keywords: mobile devices, IoT, system security, threats

JEL classification: O30, O33, O39

DOI: $10.12948 / \mathrm{ie} 2019.06 .05$

\section{Introduction}

Nowadays mobile devices capture more and more areas and are used in many activities [1] like administration, health, construction, tourism and many others. But the usage it is not limited to different areas of expertise, every person in the world who possess a smart device has used it for personal needs other than calling or texting. We use these devices to do shopping for us or to buy tickets to a spectacle or any other kind of event but many of this people don't know that these facilities comes with a cost, and that cost is our privacy and the fact that we give our personal data to them. The problem is that these devices are not very secured, and they can be attacked in different ways.

A threat refers to anything that has the potential to cause serious harm to a mobile system. A threat is something that may or may not happen but has the potential to cause serious damage. When someone breaks into a mobile system, that person takes advantage of multiple factors like technology, lapses in procedures or management (or some combination of them), allowing unauthorized access or actions. The specific failure of the controls is called a vulnerability or security flaw [2].

When we talk about any newly discovered incident that can harm a mobile device we talk about mobile security threats and when we talk about vulnerabilities we talk about some already known weakness of an asset which can be exploited by attackers [3].

The usage of mobile devices (smartphones, tablets, wearables or IoT) have increased a lot in the past years. Over the last 5 years, their numbers have grown significantly and are expected to grow even further over the next 5 years.

As we see in Figure 1, the number of IoT connected devices is nearly double in 2019 compared to 2015, it will double in 2020 and will be 5 times higher over 6 years, in 2025 . This enormous increase it happens in only then years, and this only for the IoT devices which are just a part of the mobile devices. For example, if we will look at the numbers of the smartphone users from 2014 until 2020 from Figure 2 we'll see that within 6 years the numbers is almost double. 


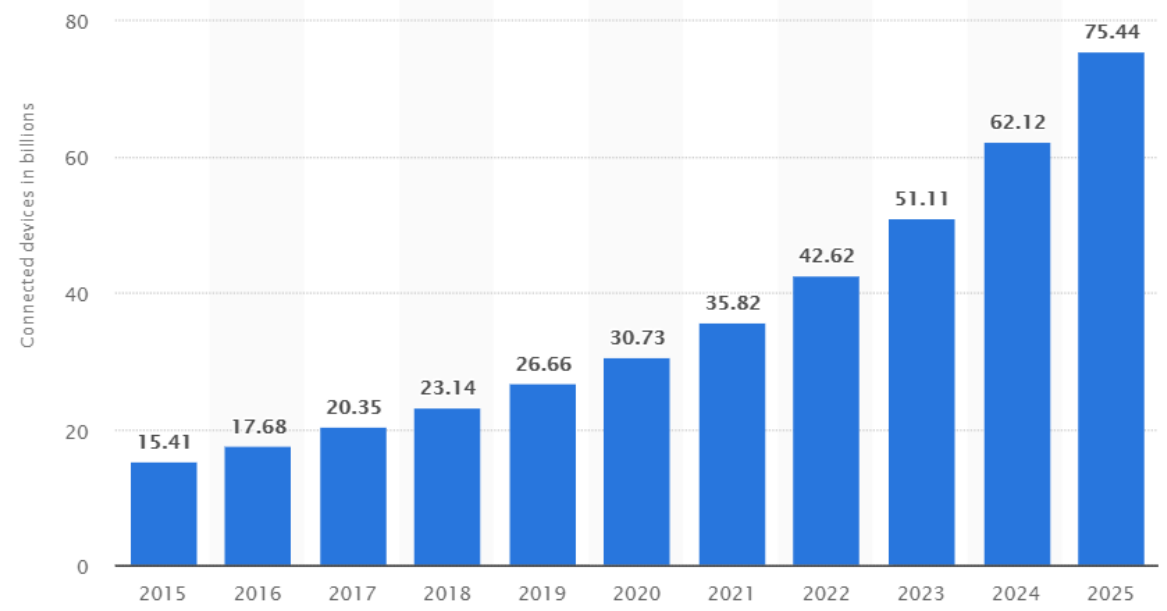

Figure 4. IoT connected devices installed base worldwide from 2015 to 2025 [4]

About 3 billion people, one third of the people in the world, have a smartphone [4].

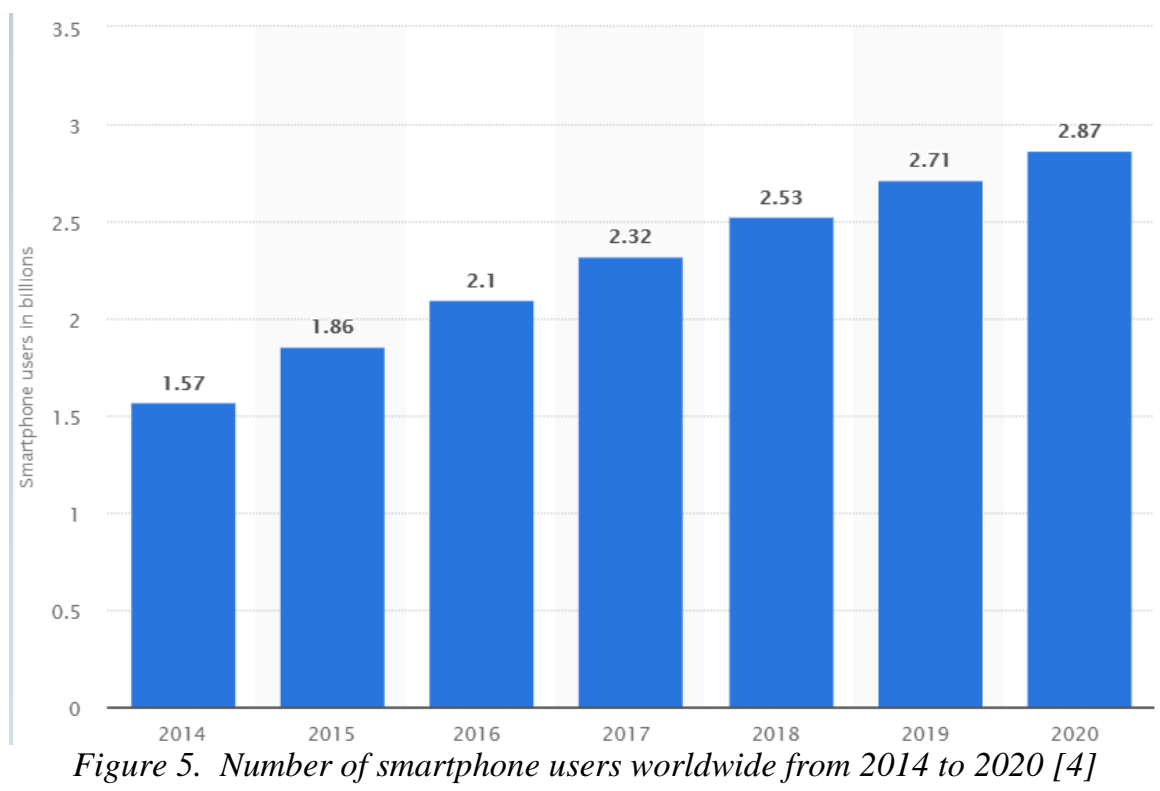

The reason these smart devices appeared was the need for people to connect to the Internet more easily and in multiple places. Today, however, they do much more than just connect to the Internet and even more than monitor heartbeat or sleep time [5]. We use these devices to connect with people everywhere, to pay bills, shop, rent cars or houses, take a ticket to the theater or a plane ticket, we connect to our bank accounts via those devices and much more. The usage of IoT is way wider from the old and know smart home to the smart car, smart farming, smart city and smart anything [6], even smart sneakers. Lenovo team made a pair of smart sneakers that can show the mood of the ones who wear them [7].

As we seen the number of mobile devices is big, the possibilities for usage are many and because of that we have to give them personal data. This comes with a great risk for user's privacy and can lead to many security threats [5]. But what exactly are those threats? How they appear? Are all the threats the same? How these vulnerabilities occurred? Can we prevent 
them? Nick Dawson, global director of enterprise business of Samsung Electronics said: "mobile security has never been a more urgent concern than it is today, and the threat defense landscape continues to evolve at an accelerating peace".

The paper is structured as follows. The second part is an overview of the mobile threats and vulnerabilities. The focus here is on smartphones and tablets but it is presented a classification for the IoT threats too, a detailed one is planned to be made in the future. The third section presents a comparison between Android and iOS threats. The paper ends with conclusion that will leave us with some questions and some plans for future work.

\section{Mobile and IoT threats and vulnerabilities}

\subsection{Mobile devices}

These threats are not only software based, they are also physical. As we mentioned above these mobile devices are used more and more for a lot of activities from which a part of them require users personal data like personal bank account and many others [8]. All of this data and the fact that, at least in case of the smartphones, there are much complex functions that a mobile device is capable, which come with a pretty much financial cost, make these devices very valuable targets and they are easy to be stolen or lost [9]. Mobile device vulnerabilities exist in the wireless connection, the device itself, a user's personal practices the organization infrastructure and wireless peripherals (keyboard, printers, mouse) which contains software, an OS and data storage device [10].

So far we can group these mobile threats as physical threats and software-based threats.

Physical threats and vulnerabilities include lost device, damaged device and stolen device or how the user use it. Physical threats have a simple resolve but very hard to put in practice. To eliminate much of this kind of threat, we need to educate ourselves and those around us that we are no longer so greedy.

Software-based threats and vulnerabilities occur at the application level, network level or device level (or OS threats) [11]. For example, at the application level, a device can be attacked through a SMS Trojan and the attacker can get money from it, or when the device it is connected to some unsecured $\mathrm{Wi}-\mathrm{Fi}$, it can leak medical results or other important data. A vulnerable Wi-Fi connection is an unencrypted one, which become a "secure" way to leak data. The point here is that exists many ways through which attackers can get from devices data like credit card details, transactions, different accounts, call logs and many others without the user's permission in order to harm him or to use that data for personal benefits. At the application level can be a more vulnerabilities like incorrect permission settings, potential functionalities that can access user personal resources and many others [10].

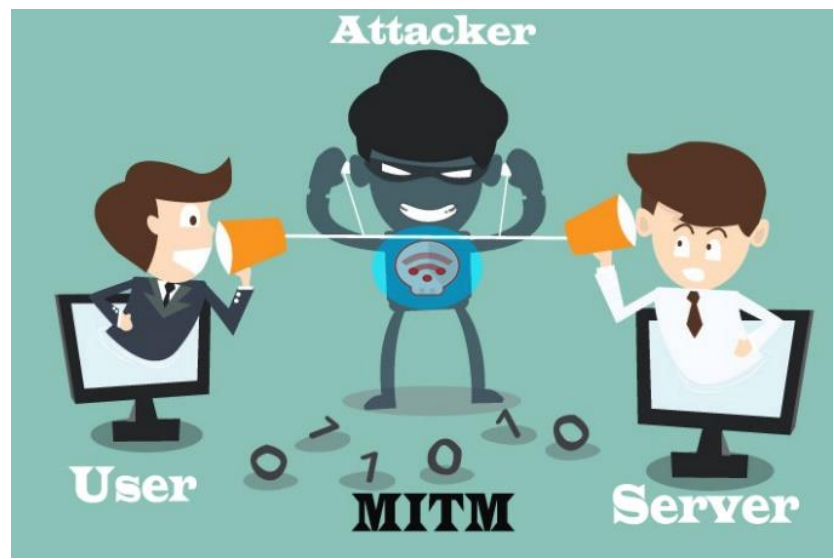

Figure 3. Man in the Middle Attack 
When we talk about threats at the network level we talk about man-in-the-middle or phishing/smishing or rogue cell tower or others. Man in the middle appear when a third entity alters a communication between other 2 entities (see Figure 3 ) and this usually happens through unprotected Wi-Fi hotspots [12].

Rogue cell towers is a cell tower operated by some rogue individuals who can trick the mobile devices making them believe that the tower is trusted and secured. Once the device is connected to the rogue tower they have access at the data sent via network, at SMS or calls [12].

Phishing/smishing were attacking only PC's until a year or two ago, but now they widen their targets and the number of attacks is increasing at a high speed. The users are trapped in this kind of threat when they click on untrusted and/or malicious links, pop-ups or e-mails that were spoofed as shown in Figure 4. The number of this attack is rising because the cost for the cybercriminal is low and it is very effective [12].

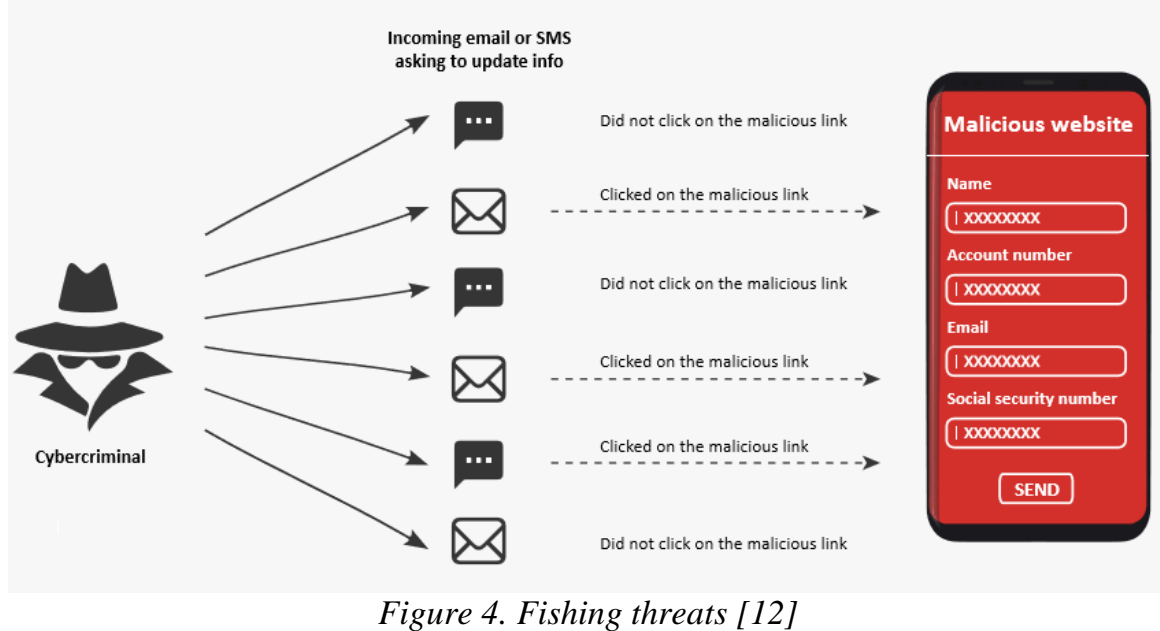

At the device level we can talk about OS vulnerabilities exploit, or jailbroken device exploit or unmanaged/malicious profile or others. An unmanaged profile can lead to exposing the data through a network very easy, jailbreak will remove the device security checks and limitations and this way will ease the access to the data. OS security vulnerabilities are some holes discovered by some malicious entities which will use it for personal benefits [11]

\subsection{IoT systems}

An IoT system is formed from many components at many levels (see Figure 6), but the most important ones are sensors/devices, connectivity, data processing and user interface. The main responsibility of sensors is to collect data, data that can came in different form, from temperature to a video feed. A device can have one or more sensors which can "work" together to do more than just sense things. For example, the smartphone has a GPS sensor, an accelerometer sensor that can be used to get data about distance and speed. Now that we collected the data we have to send it to the cloud so it can be processed. The possibilities of connecting the sensors to the cloud are multiple, like via Wi-Fi, Bluetooth, cellular/satellite networks or WAN and etc., but every possibility comes with his pluses and minuses so we should choose wisely. Once the collected data gets to the cloud it need to be processed. How the data is processed depends on its type, for example if it is about temperature it only need to be read. Once the data is processed, it has to be displayed to the user. The user can be announced about modifications through triggers, alarms or any other ways [14]. 


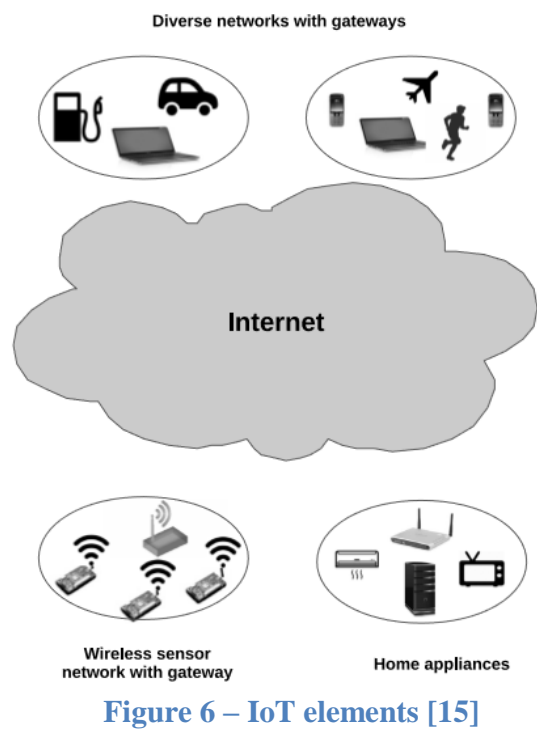

Based on this specific architecture of IoT systems, we could talk about 3 categories of security issues [15]:

- Low-level security issues

- Intermediate-level security issues

- High-level security issues

The low-level security issues occur at the hardware level and data and link layers of communication. Some of these threats are insecure initialization and insecure physical interface. The intermediate-level security issues appear at network and transport layers and affect the communication, routing and session management while the high-level security issues are mainly concerned with the applications executing on IoT [15].

\section{Case Study: Android vs iOS}

The Android system is more exposed to threats than iOS system because there are way more devices on Android than on iOS. Also the iOS system is built to be more restrictive and secure than the Android system. As we see in Figure 7 the rate of threats is higher on Android for all those 4 commences, as expected. Data leakage appears in both platforms, on Android with a proportion of $61 \%$ that is almost double of the proportion that appears on iOS. When we talk about network exploits the Apple platform is obviously more secure than the one from Google with only $1 \%$ of attacks compared to the $13 \%$ of the Android system, but here we must not forget the numbers of user's on those two platforms. Overall, from the figure bellow results that the iOS platform is more secure.

The software-based threats have a very high occurrence at the application level as we seen in the Figure 8. 
www.conferenceie.ase.ro

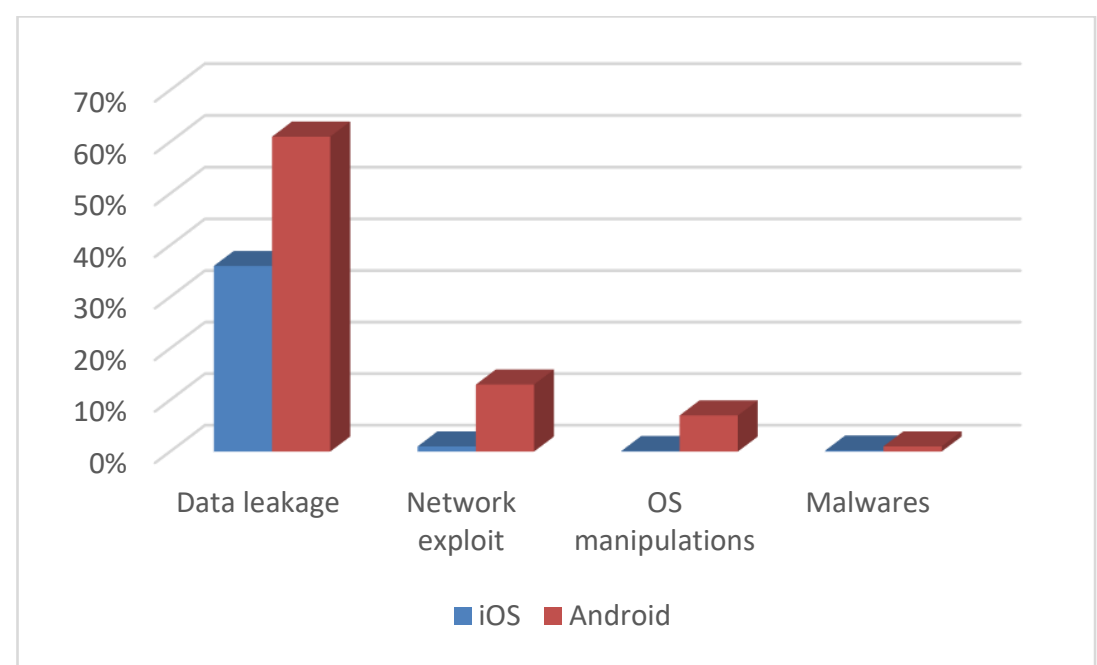

Figure 7. Threats percentage on Android vs iOS [12]

Data exfiltration is the most common threat at the application level in the last 2 years, but let us not forgot about malwares, which even their number decreased, they are still extremely dangerous [11]. So the most harmful are the leaky applications and malwares.

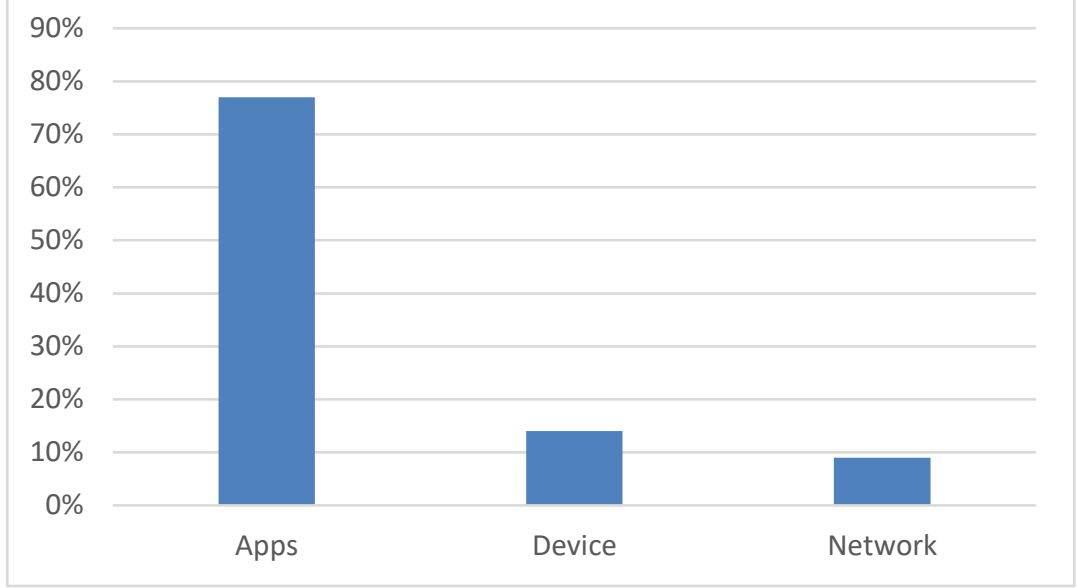

Figure 8. The mobile attack surface [11]

Now let us see the percentage of hacks on free vs paid apps on those two platforms (see Figure 9). Despite the platform, either Android either iOS, both have allowed application that can be hacked. Again, the higher proportion of attacks is registered by the Android platform, but in this case the difference it is not big, it is $10 \%$ for the paid apps and only $5 \%$ for the free apps. It is understandable why paid apps are more attacked than the free ones; a paid app has a higher chance to contain card details or data regarding bank accounts than the others.

For example, Pegasus is a spyware discovered in an iOS system version that was used for tracking calls, collecting information from application, tracking device location, collecting passwords and other actions harmful for the user. 


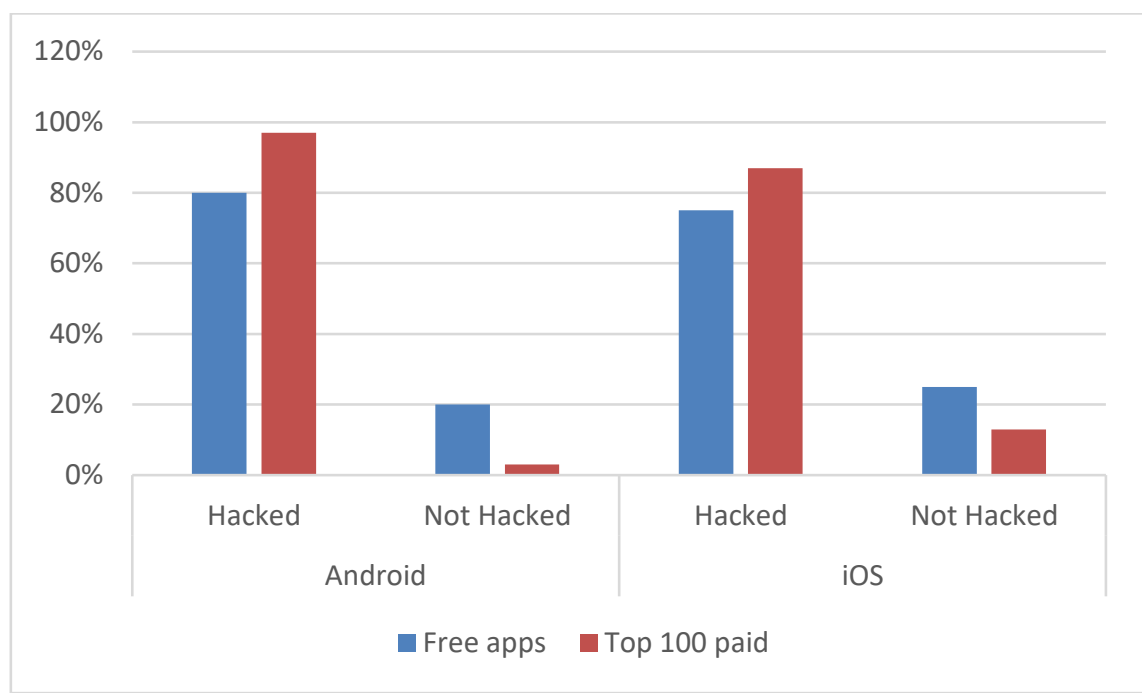

Figure 9. Percent of hacks free vs paid apps [16]

As we seen in Figure 7 and 9 there are vulnerabilities, even if they are in different proportions still they exist, in both systems. Yes, the iOS system is rumored to be more secured than Android but it also has threats and that it is not something we as users want.

Table 1. Android vs iOS Threats comparison

\begin{tabular}{|l|c|c|}
\hline Threat & Android & iOS \\
\hline Accidental Data Leakage & $\mathrm{X}$ & $\mathrm{X}$ \\
\hline Monetary Theft & $\mathrm{X}$ & $\mathrm{X}$ \\
\hline Data Exfiltration & $\mathrm{X}$ & $\mathrm{X}$ \\
\hline Network Exploit & $\mathrm{X}$ & $\mathrm{X}$ \\
\hline Malwares & $\mathrm{X}$ & $\mathrm{X}$ \\
\hline OS Exploits & $\mathrm{X}$ & $\mathrm{X}$ \\
\hline Hardware vulnerabilities & $\mathrm{X}$ & $\mathrm{X}$ \\
\hline Jailbreak & $\mathrm{X}$ & \\
\hline Hidden Root & & \\
\hline
\end{tabular}

Table 1 presents some of the most present threats in our days in order to see which of those two systems have or have not one of these vulnerabilities. From what we see, none of the system is threat free, and the worst part is that both are vulnerable to multiple kinds of attacks. We can say that the iOS system may be more secured than the Android system or vice versa but the real problem is that neither of them is $100 \%$ secure.

\section{Conclusions}

Mobile threats appear in many ways, because of different reasons, reasons according with each platform or system either Android, or iOS or IoT or other system. It is important to know what they do and how they appear so we can find ways to prevent them or as the last instance to destroy them if possible. We discussed about a classification on multiple layers and from two points of view, the physical one and the system-based one. We how this threats attack, but I think we should go this research to the smallest level possible. This classification can be taken to an even more granular level for each system. This will be a subject for future work along with analyses regarding some of the most critical threats. An application that will help in detecting at least a part of these threats on other application or to tell how secure or insecure is will be also part of the future work. 
www.conferenceie.ase.ro

\section{References}

[1] I. Adascalitei and M. I. C. Baltoi, "The influence of Augmented Reality in Construction and Integration into Smart City," Informatica Economica, vol. 22, no. 2, pp. 55-67, 2018.

[2] M. Bishop, Introduction to computer security, Boston: Pearson Education, Inc, 2004.

[3] S. Watts, "https://www.bmc.com," bmc, 21 June 2017. [Online]. Available: https://www.bmc.com/blogs/security-vulnerability-vs-threat-vs-risk-whats-difference/. [Accessed 12 March 2019].

[4] Statista, "https://www.statista.com," Statista, 2008. [Online]. Available: https://www.statista.com/statistics/471264/iot-number-of-connected-devices-worldwide/. [Accessed 26 February 2019].

[5] P. Abhishek and W. Zhiwei, "Introduction to the Special Section on Challanges and Solutions in Mobile Systems Security," Computers \& Electrical Engineering, no. 59, pp. 201-203, 2017.

[6] R. Margaret, R. Linda, S. Sharon and W. Ivy, "https://internetofthingsagenda.techtarget.com," IoTAgenda, 2016. [Online]. Available: https://internetofthingsagenda.techtarget.com/definition/Internet-of-Things-IoT. [Accessed 26 February 2019].

[7] B. Heater, "https://techcrunch.com," TechCrunch, 2016. [Online]. Available: https://techcrunch.com/2016/06/09/lenovo-smart-shoes/?guccounter=1. [Accessed 26 February 2019].

[8] F. Stroud, "https://www.webopedia.com," Webopedia, [Online]. Available: https://www.webopedia.com/TERM/M/mobile_security_threats.html. [Accessed 228 February 2019].

[9] R. Paul and F. Jon , "Cyber Threats to Mobile Phones," Carnegie Mellon University, Pittsburgh, 2011.

[10] L. G. Wlosinski, "Mobile Computing Device Threats, Vulnerabilities and Risk Factors Are Ubiquitous," ISACA, vol. 4, no. 1, pp. 1-5, 2016.

[11] Pradeo Lab, "Mobile threat report," Pradeo, 2018.

[12] Pradeo Lab, "iOS Security Report," Pradeo, 2018.

[13] Pradeo Lab, "Mobile Security Report," Pradeo, 2019.

[14] Dataflair team, "https://data-flair.training," Dataflair team, 1 June 2018. [Online]. Available: https://data-flair.training/blogs/how-iot-works/. [Accessed 8 April 2019].

[15] A. K. Minhaj and S. Khaled, "IoT security: Review, blockchain solutions and open challenges," Future Generation Computer Systems, no. 82, pp. 395-411, 2018.

[16] N. Agarwal, "Android vs iOS: Which Platform is More Secure in 2018," 1 February 2018. [Online]. Available: https://appinventiv.com/blog/android-vs-ios-which-platform-ismore-secure-in-2018. [Accessed 25 March 2019]. 